investigations (eg, enteroscopy, gastroscopy and colonoscopy), 52\% underwent therapeutic intervention (eg, APC, polypectomy and surgical referral). However, in $30 \%$ their management was unchanged. The majority of patients (88.1\%) are still under active follow-up today.

Conclusion The CE experience at this centre is of a simple, welltolerated investigation which allows definite diagnoses to be made with minimal complications. Higher diagnostic yield compared to previous published data could be explained by the strict inclusion criteria and recent introduction of this new service. This expanding service highlights the need for more resources to reduce waiting and reporting times in line with other GI investigations.

Competing interests None declared.

\section{PTU-163 DEPRESSION AND CARCINOID SYNDROME: IS THERE ANY RELATIONSHIP? A CROSS-SECTIONAL STUDY}

doi:10.1136/gutjnl-2012-302514c.163

1J Garcia-Hernandez, ${ }^{1} \mathrm{M}$ Mohmaduvesh, ${ }^{1} \mathrm{P}$ Davies, ${ }^{1} \mathrm{C}$ Toumpanakis, IJ R Goodhand, 'M Caplin, ${ }^{2} \mathrm{D}$ Skuse. ${ }^{1}$ Royal Free Hospital, London, UK; ${ }^{2}$ UCLH, London, UK

Introduction The relationship between brain serotonin and depression is well established. It is also widely accepted that serotonin hyperproduction in carcinoid syndrome does not cross the brain barrier. CNS serotonin is synthesised from tryptophan within serotonergic neurons and a deficiency of this precursor could be possible on carcinoid patients. In this cross-sectional study we evaluated, whether the feelings of depression are associated with neuroendocrine symptomatology. In addition, whether self-report of quality of life is associated with feelings of depression.

Methods 47 patients with carcinoid syndrome completed a survey via clinic involving: QoL questionnaires and the Beck's Depression Inventory-II (BDI-II). All questionnaires were counterbalanced.

Results 45 out of 47 patients had low scores on the BDI-II and not likely to suffer from depression. Only two participants had moderate depression scores. These scores were negatively associated with self-report of QoL, $r=-0.59, \mathrm{~N}=46, \mathrm{p}<0.001$. Interestingly, endocrine symptoms that is, flushing and night sweats, were not associated with depressive symptoms: $\mathrm{r}=0.22, \mathrm{~N}=44, \mathrm{p}=0.15$, whereas, gastrointestinal symptoms that is, diarrhoea, abdominal discomfort, bloatedness and indigestion, were strongly associated with depression scores: $r=56, N=43, p<0.001$.

Conclusion Depressive scores in carcinoid patients are often attributed to the stress of diagnoses and adaptation to this chronic disease, however, although a causal relationship has not been established between carcinoid and depression, physicians should consider this possibility when psychological symptoms do not improve with conventional therapy. Further research is needed to understand why gastrointestinal, and not endocrine, symptoms were correlated with depressive symptoms.

Competing interests None declared.

\section{REFERENCES}

1. Lapeire LD, Tansens A, Lemmens G, et al. Carcinoid encephalopathy: A single entity or a spectrum of different disorders. Acta Oncologica 2010;49:268-70

2. Major LF, LaVonne Brown G, Wilson WP. Carcinoid and psychiatric symptoms. South Med J 1973:66:797-90.
3. Rosenthal MA. Carcinoid associated encephalitis successfully treated with tryptophan. J Clin Neurosci 2004;11:66-7.

4. Williams MD, Dolenic T. Selective serotonin reuptake inhibitors and patients with carcinoid tumor. Psychosomatics 2005:46:370-2.

\section{Oesophageal I}

\section{PTU-164 THE CLINICAL UTILITY OF THE ENDOSCOPIC FUNCTIONAL LUMINAL IMAGING PROBE IN EOSINOPHILIC OESOPHAGITIS: A CASE SERIES}

doi:10.1136/gutjnl-2012-302514c.164

${ }^{1}$ A Simpson, ${ }^{* 1} \mathrm{M}$ S J Wilson, ${ }^{2}$ A Ellefson, ${ }^{1}$ S Colley, ${ }^{1} \mathrm{~S}$ E Attwood. ${ }^{1}$ Department of Surgery, North Tyneside General Hospital, North Shields; ${ }^{2}$ Ardmore Healthcare Limited, Amersham, UK

Introduction Disease severity assessment in eosinophilic oesophagitis (EoE) is limited by the poor correlation of endoscopic appearance and histological eosinophil density with symptoms. Our aim is to ascertain whether having a measurement of oesophageal wall distensibility (using the EndoFLIP device) helps clinical decision making in the management of patients with EoE.

Methods Dysphagic patients with proven/suspected EoE were assessed by upper gastrointestinal endoscopy, oesophageal biopsies, clinical history, weight, and medication history. EndoFLIP assessment was carried out if symptoms persisted despite medical treatment or if there was an uncertain diagnosis. Assessment was performed under general anaesthetic. The $8 \mathrm{~cm}$ EndoFLIP balloon was inflated in the lower oesophagus, first to 20 , then 30 and up to $40 \mathrm{ml}$ of water according to oesophageal wall distensibility. After balloon emptying and repositioning in the upper oesophagus, the measurements were repeated. Oesophageal diameter was recorded at $5 \mathrm{~mm}$ intervals and distensibility calculated as changes in crosssectional area per $\mathrm{mm} \mathrm{Hg}$. If the lumen was poorly distensible and $<11 \mathrm{~mm}$ across, a through-the-scope balloon dilator was used to expand the areas depicted by EndoFLIP as being poorly compliant. Repeat EndoFLIP testing immediately after dilation revealed the improvement in distensibility. Patients were followed up at 3 months.

Results 6 patients in total were included, five of whom had biopsy proven EoE. In all six patients we found the EndoFLIP useful in guiding management. Two of the five patients with EoE were found to have poor oesophageal distensibility and underwent dilatation, following which a clear improvement in distensibility was seen. This correlated with an improvement in symptoms at follow-up. The remaining three patients with proven EoE had normal distensibility measurements and therefore did not undergo dilatation as a result of EndoFLIP testing. The single patient without EoE had normal distensibility measurements. Dilatation was undertaken following tertiary centre consultation on the basis of high-resolution manometry testing but symptoms returned after 3 months. There were no difficulties in performing the EndoFLIP test. There was a single complication of oesophageal mucosal tear following dilatation, which was of no clinical significance.

Conclusion Measuring the oesophageal wall distensibility may become a useful tool in the clinical assessment of EoE and may help to define the need for oesophageal dilatation and predict the outcome of such intervention.

Competing interests A Simpson: None declared, M Wilson: None declared, A Ellefson Employee of: Ardmore Healthcare Limited, S Colley: None declared, S Attwood: None declared. 


\section{REFERENCE}

1. Kwiatek MA, Hirano I, Kahrilas PJ, et al. Mechanical properties of the esophagus in osinophilic esophagitis. Gastroenterology 2011;140:82-90.

\section{PTU-165 ENDOSCOPIC RESPONSE OF OESOPHAGEAL CARCINOMA TO NEO-ADJUVANT CHEMOTHERAPY AND SURVIVAL POST-OESOPHAGECTOMY}

doi:10.1136/gutjnl-2012-302514c.165

A M Jones, ${ }^{*}$ J Cornish, D Chan, G Clark. Upper Gastrointestinal Surgery, University Hospital of Wales, Cardiff, UK

Introduction Endoscopy and biopsy is an essential initial investigation in oesophageal carcinoma. At our unit a repeat endoscopy is conducted after a patient has completed neo-adjuvant chemotherapy and prior to proceeding to oesophagectomy, predominantly to obtain anatomical information. This study aims to determine whether prognostic information can be derived from the response to chemotherapy.

Methods Data were retrospectively collected for patients who had undergone oesophagectomy following neo-adjuvant chemotherapy under a single surgeon over a 10-year period. At endoscopy after chemotherapy, it was noted whether the tumour had made a complete response, a partial response or no response at all, according to length, circumferential involvement and degree of stenosis. Survival data were obtained and compared between groups.

Results 72 patients (60 Male, 12 Female) were identified. 25 patients had no response to neo-adjuvant chemotherapy as assessed endoscopically and had a median survival of 27 months and 2-year survival of $38 \%$. 39 patients showed a partial response endoscopically and had a median survival of 30 months and a 2-year survival of $40 \%$. For the eight patients who had a complete response median survival was 57 months and 2-year survival of $68 \%$.

Conclusion The response to neo-adjuvant chemotherapy as assessed endoscopically is predictive of post-operative prognosis. A larger study is required to determine if endoscopic assessment could be used to select patients unlikely to benefit from surgery.

Competing interests None declared.

\section{PTU-166 COMPARATIVE OUTCOMES FOLLOWING SURGERY FOR PATIENTS ENTERING OEO2 AND MAGIC NEO-ADJUVANT REGIMENS}

doi:10.1136/gutjnl-2012-302514c.166

${ }^{1}$ A Reece-Smith, ${ }^{2}$ J P Duffy, ${ }^{2}$ S Madhusudan, 'S L Parsons. 'Oesophago-Gastric Surgery, Nottingham University NHS Trust, Nottingham, UK; ${ }^{2}$ Department of Medical Oncology, Nottingham University NHS Trust, Nottingham, UK

Introduction $\mathrm{MAGIC}^{1}$ regimen of three cycles of ECF chemotherapy pre- and post-op is widely used in gastric cancer but its use in oesophageal cancer remains controversial. We have compared post operative survival outcomes of oesophageal and GOJ cancer patients entered into either $\mathrm{OEO}^{2}$ (2 cycles of CF pre-operatively) or MAGIC regimen to determine if either is advantageous.

Methods A database of cancer resections was maintained from 2000 until present. Patients fit for treatment were mainly given OEO2 regimen from 2002 to 2006 and MAGIC regimen from 2006-present. The database was searched for patients receiving pre-operative chemotherapy using either ECF/X or CF/X. Kaplan-Meier survival analysis was undertaken using log-rank test for comparisons.

Results Median follow-up was more than 8.5 years for the 97 patients with complete data in the OE02 group and 3.7 years for the 138 complete patients in the MAGIC group. Oesophageal cancer patients that had received MAGIC regimen pre-operatively had significantly longer median survival compared to those that had received OE02 (34.0 months vs 23.4 months, $p=0.033$ ). A significant benefit was not shown in GOJ cancer (MAGIC: 32.3 months, OE02: 23.5 months, $\mathrm{p}=0.095$ )

Conclusion Oesophageal cancer patients attending for curative surgery that commenced treatment with MAGIC style chemotherapy have better survival than patients that started OE02 regimen

Competing interests None declared.

\section{REFERENCES}

1. Cunningham, et al. NEJM 2006;355:11-20.

2. MRC Working Party. Lancet 2002;359:1727-33.

\section{PTU-167 THE ROLE OF PROTON PUMP INHIBITORS IN PATIENTS WITH BARRETT'S OESOPHAGUS FOLLOWING LAPAROSCOPIC FUNDOPLICATION CAN ONLY BE DETERMINED AFTER THE LEVEL OF RESIDUAL REFLUX HAS BEEN QUANTIFIED}

doi:10.1136/gutjnl-2012-302514c.167

A Bond, ${ }^{*}$ D Wilson, A Hastings, F Rowley, D Veeramootoo, K K Singh. Department of Surgery, Worthing Hospital, Worthing, UK

Introduction Laparoscopic fundoplication is carried out for pathological Gastro Oesophageal Reflux in patients with and without Barrett's oesophagus. It has been suggested that in patients with Barrett's, only those patients with no physiological evidence of reflux post surgery have any reduction in risk of transition to cancer. The aim of this study is to quantify and compare residual reflux post fundoplication in patients with and without Barrett's Oesophagus. Methods Data were collated from January 2002 to December 2011, using the hospital coding database for a single consultant surgeon. Each case was studied for patient demographics, indication, operative findings and outcomes. Prospectively collected data on pre and post operative $\mathrm{pH}$ manometry was assessed for evidence of reflux. Total percentage time less than $\mathrm{pH} 4$ was used to determine reflux and a paired t-test was used to compare reflux between the groups. Results 78 patients had a Nissen Fundoplication, 32 with Barrett's oesophagus. There was no significant difference between age and sex of the Barrett's and non Barrett's groups. There is seen to be a significant difference in reflux between the pre operative groups $(p=0.0227)$ when looking at reflux, but a very significant difference $(p=0.0038)$ when comparing reflux postoperatively between those with and those without Barrett's.

Conclusion There is a significantly higher level of residual reflux in patients with Barretts oesophagus following fundoplication even when patients report no symptoms. Therefore those with Barrett's should continue with Proton Pump Inhibitors until there is confirmed evidence of no reflux.

Competing interests None declared.

\section{PTU-168 2-STAGE OESOPHAGECTOMY CONFERS NO SURVIVAL ADVANTAGE OVER TRANSHIATAL RESECTION: ANALYSIS OF 550 CONSECUTIVE CASES IN A SINGLE UNIT}

doi:10.1136/gutjnl-2012-302514c.168

A Davies, ${ }^{*}$ H Sandhu, A Pillai, P Sinha, S Helme, J Deguara, J Gossage, A Botha, R Mason. Surgery, St Thomas' Hospital, London, UK

Introduction The optimal operative approach to resectable cancers of the oesophagus and oesophago-gastric junction is contentious. Both 